\title{
Immunotherapy of Peritoneal Carcinomatosis with Catumaxomab
}

\author{
Simon L. Parsons \\ Nottingham University Hospitals NHS Trust, UK
}

The study by Ströhlein et al. [1] in this issue of ONKOLOGIE evaluates a new potential treatment for peritoneal carcinomatosis (PC). PC is a significant problem in gastrointestinal (GI) cancer, particularly gastric cancer. It is caused by the transcoelomic spread of cancer and can occur with or without evidence of metastasis via other routes. It is a sign of advanced malignancy and is usually associated with a poor prognosis commonly progressing to bowel obstruction or malignant ascites [2]. There have been a number of staging systems for classifying PC but the most commonly used is that described by Gilly and co-workers that classifies PC by size of nodules and distribution within the peritoneal cavity [3]. This staging system was used in the EVOCAPE 1 study by Sadeghi and colleagues where 370 patients were followed after diagnosis of PC at open surgery and was shown to be an important prognostic indicator [2]. 22 out of 24 patients in Ströhlein's study had advanced PC according to the Gilly classification and therefore would be expected to have a poor survival.

The prognosis of patients with PC secondary to GI cancer is poor: in the EVOCAPE 1 study the mean and median survivals were 6 and 3.1 months, respectively. Median survival is tumour dependent with the main tumour groups of gastric, colorectal and pancreatic cancers having median survivals of 3.1, 5.2 and 2.1 months respectively. An earlier study, by Chu and colleagues, of 100 patients were prospectively studied with colorectal cancer being the most common primary tumour and both gastric and pancreatic cancers being associated with the worst prognosis particularly when associated with ascites [4].

In the laparoscopic era, staging laparoscopy is commonly performed in upper GI cancers to prevent unnecessary open and close procedures in patients with PC secondary to gastric, pancreatic and oesophageal adenocarcinomas. A recent study looking at survival of gastric and oesophageal cancer patients found to have PC at laparoscopy who did not proceed to surgical resection showed that patients with overt PC survived a median of 9 months when receiving chemotherapy compared to 6 months in those who did not [5].

\section{KARGER \\ Fax +497614520714 \\ Information@Karger.de}

www.karger.com (c) 2011 S. Karger GmbH, Freiburg

0378-584X/11/0343-0086\$38.00/0

Accessible online at:

www.karger.com/onk
Peritonectomy and cytoreductive surgery with or without HIPEC (hyperthermic intraperitoneal chemotherapy) has been performed with some impressive results in patients with PC in slow growing GI tumours [6]. Patients with PC secondary to gastric cancer have been shown to have a median survival of up to 12 months with complete cytoreduction [7]. However, complete cytoreduction is often not possible and this technique carries significant morbidity and mortality. Thus non-operative techniques which give a similar median survival are preferable. The median survival from diagnosis in the study by Ströhlein et al. [1] was 16.7 months for the catumaxomab group which compares very favourably with their control group (6 months) and with the literature.

Catumaxomab is a monoclonal antibody targeted against epithelial cell adhesion molecule (Ep-CAM) on the tumour cell which is expressed on over $90 \%$ of epithelial cancers [8]. This high expression makes such a target very attractive especially compared to other biological targets. Ep-CAM is expressed on normal epithelial cells baso-laterally and is shielded by tight junctions that limit its accessibility [9]. Due to the fact that the peritoneal cavity is lined with mesothelium, the only cells expressing Ep-CAM in the peritoneal cavity will be tumour cells, thus making the intraperitoneal route ideal for administration of catumaxomab and PC the ideal disease target. The decrease in the number of Ep-CAM positive tumour cells in the peritoneal lavage samples and the lack of progression to malignant ascites, give additional evidence of tumour response in the current study.

Catumaxomab does cause a cytokine release syndrome with activation of IL-6, TNF $\alpha$ and other cytokines. This explains the majority of adverse events seen, which are broadly similar to that seen in the phase II/III randomized trial of malignant ascites [10] despite the slightly higher final dose in this PC study. One of the main factors limiting the use of catumaxomab in the malignant ascites trial was that the condition of many patients had deteriorated such that their Karnovski performance score was less than 60 by the time they had completed conventional chemotherapy and had reaccumulated 
their ascites. This meant that such patients were ineligible for the trial and those that were eligible were at the most advanced stage. Patients with PC without ascites are earlier in their disease process and therefore should have a higher performance score and be better able to tolerate the cytokine release syndrome. Furthermore, they are more likely to have an intact immune system and are therefore more likely to benefit clinically as the unique mechanism of action of catumaxomab is based on recruiting both the host's T-cell and B-cell immune systems.

In summary the findings of Ströhlein and colleagues demonstrate clinical efficacy and tolerable toxicity in patients with PC secondary to GI cancer. The design of the study may give rise to selection bias and so the only way of conclusively demonstrating efficacy is in a randomized trial. This has already been shown in the more advanced setting of malignant ascites and such a randomized trial is planned in patients with PC secondary to gastric cancer comparing catumaxomab plus cisplatin and 5-fluorouracil/capecitabine versus cisplatin and 5-fluorouracil/capecitabine alone. Recruitment is due to commence in the second quarter of 2011.

\section{Disclosure Statement}

Simon Parsons sits on a malignant ascites advisory board sponsored by Fresenius Biotech and has offered advice as part of a consultancy agreement with Fresenius Biotech. He has also received research funding in the past from Fresenius Biotech. He has no shares or other financial interest in Fresenius Biotech.

\section{References}

1 Ströhlein MA, Lordick F, Rüttinger D, Grützner K-U, Schemanski OC, Jäger $\mathrm{M}$ Lindhofer H, Hennig M, Jauch K-W, Peschel C, Heiss MM: Immunotherapy of peritoneal carcinomatosis with the antibody catumaxomab in colon, gastric, or pancreatic cancer: an open-label, multicenter, phase I/II trial. Onkologie 2011;34: DOI: $10.1159 / 000324667$.

2 Sadeghi B, Arvieux C, Glehen O, Beaujard AC, Rivoire M, Baulieux J, Fontaumard E, Brachet A, Caillot JL, Faure JL, Porcheron J, Peix JL, Francois Y, Vignal J, Gilly FN: Peritoneal carcinomatosis from non-gynecologic malignancies: Results of the evocape 1 multicentric prospective study. Cancer 2000;88:358-363. 